\title{
Studies on Immunity in Anthrax
}

\author{
XII. Requirement for Phosphate for Elaboration of Protective Antigen \\ and Its Partial Replacement by Charcoal
}

\author{
GEORGE G. WRIGHT, LORELL H. ANGELETY, AND BEATRICE SWANSON ${ }^{1}$ \\ Medical Sciences Laboratory, Fort Detrick, Frederick, Maryland 21701
}

Received for publication 29 June 1970

\begin{abstract}
Retention of protective antigen on columns of diethylaminoethyl cellulose was inhibited by the $0.01 \mathrm{M}$ phosphate salts in the standard growth medium. Reduction in the concentration of phosphate to $0.001 \mathrm{~m}$ allowed satisfactory retention of antigen on diethylaminoethyl cellulose but decreased significantly the elaboration of antigen during growth of the cultures. Growth remained normal at phosphate concentrations as low as $0.0001 \mathrm{M}$. Inhibition of antigen elaboration in media containing reduced concentrations of phosphate was overcome by addition of $0.006 \%$ $(w / v)$ charcoal. It is suggested that antigen elaboration, like encapsulation of virulent strains, is stimulated in the presence of an adsorbent. However, a nonencapsulated strain derived from an $F$ mutant exhibited the same requirement for an adsorbent as nonencapsulated strains derived from the parent strain.
\end{abstract}

The protective antigen of Bacillus anthracis has found practical application in immunization of man against anthrax and in serological measurement of immunity $(1,2,5,16)$. Interest in the antigen also was stimulated by recognition of its role as a component of the anthrax toxin (14). Accordingly, workable procedures for preparation and isolation of protective antigen in good yield are of practical and theoretical significance.

The antigen is elaborated into the culture medium only under particular conditions of growth. Although considerable information has been reported regarding suitable media and cultural conditions, the requirements for elaboration of antigen remain essentially empirical (18). A relatively high concentration of bicarbonate is perhaps the best established requirement. The bicarbonate evidently permits release of antigen which otherwise remains associated with the cells (10). Other requirements for elaboration of antigen in addition to substances needed for growth include a utilizable carbohydrate, guanine or certain other purines, calcium ion, and certain amino acids (11).

Addition of small amounts of charcoal stimulated elaboration of antigen in hydrolyzed casein media and in certain chemically defined media (16). In unpublished experiments in our laboratory with medium 1095 and other defined media, however, no stimulation of antigen elaboration was detected on addition of charcoal. In the present work, difficulties encountered in the concen-

\footnotetext{
1 Present address: Armed Forces Radiobiological Research Institute, Bethesda, Md.
}

tration of protective antigen by chromatographic methods led to study of the effects of the concentration of phosphate in the medium and to reinvestigation of the addition of charcoal.

\section{MATERIALS AND METHODS}

Cultures. The V770-NP1-R and 116-NP1-R2 strains of $B$. anthracis were used for the production of protective antigen (18). F mutants were isolated from strain 116-NP2 by the method of Meynell and Meynell (9). Nonencapsulated mutants were obtained from the $\mathrm{F}$ mutants as described by Thorne (15).

Growth media. Medium 1095 (18) was the basal medium for production of protective antigen. Media that contained reduced concentrations of phosphate were adjusted to $p \mathrm{H} 6.4$ to 6.5 before autoclaving. After aseptic addition of all supplements, the complete medium was adjusted to $p \mathrm{H} 7.8$ to 7.9 with $1 \mathrm{~N}$ $\mathrm{HCl}$.

Vegetative inoculum. One-liter volumes of medium 1095 were inoculated with 500 spores per $\mathrm{ml}$ and incubated for 24 to $25 \mathrm{hr}$ in air at $37 \mathrm{C}$ with moderate stirring.

Preparation of protective antigen. Cultures for routine production of culture filtrate for fractionation studies were grown in 10-liter volumes of medium (18). Cultures were inoculated with 500 spores per $\mathrm{ml}$, adjusted to $37 \mathrm{C}$, and incubated under anaerobic conditions with constant slow stirring. At the time of complete utilization of glucose, usually 25 to $27 \mathrm{hr}$, the cultures were adjusted to $p \mathrm{H} 8$ and filtered through Selas filters (Selas Flotroxics, Div. of Selas Corp. of America, Spring House, $\mathrm{Pa}$.) of $\mathbf{0 2}$ or 03 porosity.

For study of the effect of alterations in the composition of the medium on growth or antigen elaboration. 
2.5-liter volumes of medium in 4-liter bottles were used. The media were autoclaved for $25 \mathrm{~min}$ at $120 \mathrm{C}$. In some cases the indicated volumes of vegetative inoculum were used rather than spore inoculum. Cultures were incubated in the same manner as the larger cultures, except that at the indicated periods samples were removed, adjusted to $\mathrm{pH} 8$, and clarified by centrifugation for estimates of antigen titer or remaining glucose. In some cases the supernatants were sterilized by passing through membrane filters of $0.45 \mu \mathrm{m}$ pore size (Millipore Corp., Bedford, Mass.).

Charcoal. Activated charcoal (Norit A) was suspended in double-distilled water at $0.125 \%$, sterilized by autoclaving, and added aseptically to the complete medium as indicated.

Glucose determinations. The glucose oxidase method (12) was employed for glucose determinations, with Glucostat (Worthington Biochemical Corp.) used as reagent.

Phosphate determinations. The method of Fiske and Subbarow (4) was used for phosphate determinations.

Assay of protective antigen. Culture filtrates were titrated for antigen activity by the complement fixation (CF) method (7) by using serum B-48. Most of the filtrates also were examined by gel diffusion to confirm the specificity of the reaction. A circular pattern was used with a central antiserum well containing serum P-8 and six peripheral antigen wells. Protective antigen lines produced by unknown samples were identified by interaction with control lines from wells containing purified protective antigen.

Antisera. Serum B-48, used for CF-titrations, was produced in a burro by four subcutaneous injections of $5 \times 10^{9}$ viable spores of $B$. anthracis, Sterne strain. In immunoelectrophoresis, the serum produced a strong line with protective antigen and two additional weak lines with concentrated culture filtrate. We are indebted to the U.S. Army Medical Research Institute of Infectious Disease for this antiserum. Serum P-8 was obtained from a pony after eight courses of immunization with protective antigen filtrate adsorbed on aluminum hydroxide. The serum produced at least 23 arcs of precipitation with concentrated culture filtrates.

Column chromatography. Diethylaminoethyl (DEAE)-cellulose was suspended as a $7.5 \%(\mathrm{w} / \mathrm{v})$ slurry in $0.5 \mathrm{~N} \mathrm{NaOH}$ and shaken at room temperature for $10 \mathrm{~min}$. Then the material was washed thoroughly with deionized water and was washed twice with $0.01 \mathrm{M}$ phosphate buffer, first at $p \mathrm{H} 6.3$ and then at $p H$ 7.2. The weight of DEAE cellulose used for preparation of columns was determined by the volume of culture filtrate to be processed, usually at a ratio of $2 \mathrm{~g}$ of DEAE cellulose per liter of undiluted filtrate. The ratio of height to diameter of the columns was approximately $2: 1$. Columns were equilibrated to $p \mathbf{H} 7.2$ by passage of $0.01 \mathrm{M}$ phosphate buffer.

\section{RESULTS}

Initial adsorption of antigen. Exploratory studies revealed the applicability of columns of DEAE cellulose for concentration and fractionation of protective antigen and other antigens in culture filtrates of $B$. anthracis. Dilution of the culture with 1.25 or 1.5 volumes of water reduced the ionic strength sufficiently so that the antigens were adsorbed on the columns. Passage of phosphate buffers containing appropriate concentrations of sodium chloride eluted the various antigens in acceptable yield.

More detailed study of the recovery of protective antigen during initial adsorption on DEAE cellulose revealed that, after passage of relatively small volumes of diluted culture filtrate, a significant proportion of the antigen remained in the effluent. The attainable loading of the column was much less than in subsequent purification steps. Typical results are presented in Fig. 1, in which the per cent of antigen adsorbed is plotted as a function of volume of filtrate passed.

A similar incomplete adsorption of antigen was observed when suspensions of DEAE cellulose were mixed with diluted culture filtrates, and this more convenient procedure was used for systematic study of factors influencing adsorption of antigen. No significant increase in adsorption was obtained by further dilution of the filtrate, alterations in $p \mathrm{H}$ in the range 7.0 to 8.8 , use of other brands or types of DEAE cellulose, alkali pretreatment or additional washing of the DEAE cellulose, change in the time of adsorption between 15 and $60 \mathrm{~min}$, or reduction in temperature to $4 \mathrm{C}$. Prior dialysis of the filtrate against water, however, greatly increased the proportion of protective antigen that combined with DEAE cellulose.

Systematic investigation revealed that the factor that inhibited quantitative recovery of the antigen was the potassium phosphate, which was present in the culture medium at a concentration

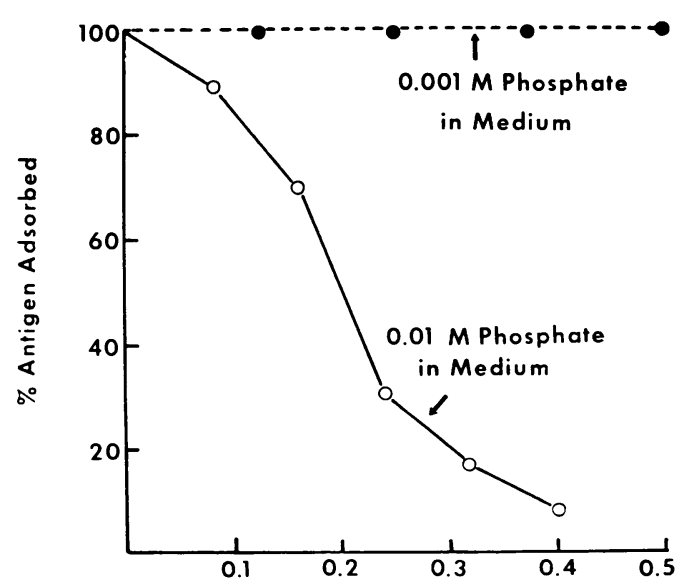

Liters of Culture Filtrate Passed per Gram DEAE

FIG. 1. Effect of concentration of phosphate in growth medium on adsorption of protective antigen from culture filtrate onto DEAE cellulose columns. 
TABLE 1. Effect of concentration of phosphate on utilization of glucose and elaboration of protective antigen

\begin{tabular}{l|c|c|c|c|c}
\hline & \multicolumn{5}{|c}{ Incubation time } \\
\cline { 2 - 5 } $\begin{array}{c}\text { Concn of } \\
\text { sodium phos- } \\
\text { phate in } \\
\text { medium (M) }\end{array}$ & $22 \mathrm{hr}$ & \multicolumn{2}{|c|}{$24 \mathrm{hr}$} & \multicolumn{2}{|c}{$27 \mathrm{hr}$} \\
\cline { 2 - 5 } & $\begin{array}{c}\text { Initial } \\
\text { glucose } \\
\text { remain- } \\
\text { ing (\%) }\end{array}$ & $\begin{array}{c}\text { CF units } \\
\text { per ml }\end{array}$ & $\begin{array}{c}\text { Initial } \\
\text { glucose } \\
\text { remain- } \\
\text { ing (\%) }\end{array}$ & $\begin{array}{c}\text { CF units } \\
\text { per ml }\end{array}$ & $\begin{array}{c}\text { Initial } \\
\text { glucose } \\
\text { remain- } \\
\text { ing (\%) }\end{array}$ \\
\hline 0.01 & 18 & 120 & 0 & 160 & 0 \\
0.001 & 21 & 40 & 0 & 40 & 0 \\
0.0001 & 25 & 30 & 0 & 30 & 0 \\
0.00001 & 82 & 15 & 83 & 15 & 81 \\
0
\end{tabular}

of $0.01 \mathrm{M}$. Reduction of phosphate in the medium to $0.001 \mathrm{M}$ or dialysis of the filtrate gave greatly increased adsorption of this antigen (Fig. 1).

Effect of phosphate concentration. The concentration of phosphate in the medium had been retained at $0.01 \mathrm{~m}$ since the initial studies on antigen elaboration in defined media (17). The phosphate was added as equimolar concentrations of dibasic and monobasic potassium phosphates. Experiments were carried out on the effect of reduction of the concentration of total phosphate on growth as estimated by the time required for complete utilization of the glucose, and on protective antigen elaboration as measured by CF titers. Typical results are presented in Table 1 . Reduction in phosphate to 0.001 or $0.0001 \mathrm{M}$ had no significant effect on rate of growth; only when the concentration was reduced to $0.00001 \mathrm{M}$ was growth delayed and decreased. Experiments on uptake of phosphate during growth were consistent with these findings; in cultures with initial phosphate concentrations ranging from 0.01 to $0.0001 \mathrm{M}$, no change in phosphate concentration could be detected after growth. Optimum elaboration of antigen required a much higher concentration of phosphate than was necessary for growth. Reduction to $0.001 \mathrm{M}$ usually reduced antigen detectably, and further reduction led to progressive loss of antigen elaboration (Table 1).

Various hypotheses were investigated in an effort to determine the basis of the requirement for $0.01 \mathrm{M}$ phosphate. Decrease in phosphate to $0.001 \mathrm{M}$ reduced the buffering power of the medium and lowered the $p \mathbf{H}$ during autoclaving from 6.8 to 3.6. However, adjustment of the medium to $p \mathrm{H} 6.8$ with $\mathrm{NaOH}$ before autoclaving did not overcome the phosphate requirement. Reduction in phosphate did not alter the $p \mathrm{H}$ during growth by as much as $0.05 p \mathrm{H}$ unit; evidently the buffering power of the complete medium results primarily from the sodium bicarbo- nate added aseptically after autoclaving. Apparently, control of the $p \mathrm{H}$ during growth was not the basis of the requirement for phosphate.

The possibility was considered that the requirement for phosphate, in addition to that necessary for growth, involved formation during autoclaving of phosphate complexes with glucose or other medium constituents that might determine the availability of critical metal ions (13). This hypothesis was discarded when it was found that phosphate or glucose could be autoclaved separately from the other constituents of the medium without significant alteration in growth or antigen elaboration.

Synthesis of alkaline phosphatase by $B$. anthracis is controlled by the concentration of phosphate in the medium (6). It seemed possible that $0.01 \mathrm{M}$ phosphate was required to repress production of alkaline phosphatase, which might be competitive with or otherwise inhibit the synthesis of protective antigen. A mutant strain was isolated in which phosphatase was repressed by $1 / 20$ the concentration of phosphate required by the parent strain. Antigen elaboration by the mutant had the same requirement for phosphate as by the parent strain; evidently, phosphatase synthesis is not involved in the requirement for phosphate.

Reduction in the concentration of phosphates influenced slightly the color of the final medium, perhaps reflecting differences in the state of colloidal phosphate salts. Optimum encapsulation of virulent $B$. anthracis requires not only bicarbonate but also an additional factor present in serum, which is replaceable by serum albumin, charcoal, or certain other adsorbents (8). It seemed possible that optimum elaboration of protective antigen might also require an adsorbent, and that the high concentration of phosphate functioned to form an adsorbent of colloidal phosphates. Accordingly, the effect of addition of charcoal on elaboration of antigen in the presence of reduced concentrations of phosphate was investigated. Preliminary experiments indicated that $0.006 \%$ $(w / v)$ charcoal was the highest concentration that did not cause marked delay in growth from spore inocula. Experiments (Table 2) explored the possible interactions of the concentrations of phosphate and charcoal during elaboration of antigen by spore and vegetative inocula of the two strains. Elaboration of antigen was inhibited by reduction of phosphate from 0.01 to $0.001 \mathrm{M}$, and this inhibition was overcome by addition of charcoal. Charcoal did not increase elaboration of antigen in media containing $0.01 \mathrm{M}$ phosphate. The requirement for $0.01 \mathrm{M}$ phosphate or charcoal appeared to be less marked when vegetative rather than spore inocula were used, especially with 
TABLE 2. Effect of concentration of phosphate and addition of charcoal on elaboration of protective antigen

\begin{tabular}{|c|c|c|c|c|c|c|}
\hline \multirow{2}{*}{ Inoculum } & \multirow{2}{*}{ Strain of $B$. anthracis } & \multirow{2}{*}{$\begin{array}{c}\text { Concn of } \\
\text { phosphate (M) }\end{array}$} & \multicolumn{4}{|c|}{ Concn of charcoal } \\
\hline & & & None & $0.002 \%$ & $0.006 \%$ & $0.019 \%$ \\
\hline \multicolumn{7}{|l|}{ Spores } \\
\hline \multirow[t]{2}{*}{$500 / \mathrm{ml}$} & V770-NP1-R & 0.01 & $80^{a}$ & \multirow{8}{*}{160} & & \\
\hline & & 0.001 & 40 & & 160 & \\
\hline \multirow[t]{2}{*}{$500 / \mathrm{ml}$} & V770-NP1-R & 0.01 & 160 & & 160 & \\
\hline & & 0.001 & 60 & & 160 & \\
\hline $500 / \mathrm{ml}$ & V770-NP1-R & 0.001 & 60 & & 160 & 160 \\
\hline $500 / \mathrm{ml}$ & 116-NP1-R2 & 0.001 & 60 & & 160 & \\
\hline $500 / \mathrm{ml}$ & 116-NP1-R2 & 0.001 & 30 & & 60 & \\
\hline $500 / \mathrm{ml}$ & 116-NP1-R2 & 0.001 & 15 & & 60 & \\
\hline \multicolumn{7}{|l|}{ Vegetative culture } \\
\hline $10 \%$ & \multirow[t]{3}{*}{ V770-NP1-R } & 0.01 & 120 & \multirow{13}{*}{320} & \multirow{4}{*}{120} & \multirow{13}{*}{240} \\
\hline $10 \%$ & & 0.001 & 60 & & & \\
\hline $0.25 \%$ & & 0.001 & 20 & & & \\
\hline $10 \%$ & \multirow[t]{2}{*}{ V770-NP1-R } & 0.01 & 320 & & & \\
\hline $10 \%$ & & 0.001 & 160 & & 320 & \\
\hline $1 \%$ & V770-NP1-R & 0.001 & 40 & & 160 & \\
\hline $10 \%$ & 116-NP1-R2 & 0.001 & 320 & & 320 & \\
\hline $10 \%$ & 116-NP1-R2 & 0.01 & 320 & & & \\
\hline $10 \%$ & & 0.001 & 240 & & & \\
\hline $1 \%$ & 116-NP1-R2 & 0.01 & 120 & & & \\
\hline $1 \%$ & & 0.001 & 60 & & 120 & \\
\hline \multirow[t]{2}{*}{$1 \%$} & 116-NP2-F-R & 0.01 & 120 & & & \\
\hline & & 0.001 & 60 & & 120 & \\
\hline
\end{tabular}

${ }^{a}$ Fifty per cent $(50 \%)$ complement-fixation units per milliliter of culture.

strain 116-NP1-R2. Titers of antigen varied somewhat among experiments, perhaps reflecting variations in sensitivity of the CF titrations, so that comparisons of titer between experiments may not be valid.

Effect of charcoal on rate of growth. Addition of $0.006 \%$ charcoal caused no significant effect on growth from vegetative inocula, as judged by the rate of utilization of glucose. With spore inocula, however, addition of $0.006 \%$ charcoal increased the time required for complete utilization of glucose by as much as $5 \mathrm{hr}$. The effect appeared to consist primarily of a lag in the initiation of fermentation. Accordingly, the influence of addition of charcoal at various times after inoculation was investigated. Typical results, showing the delay in glucose utilization when charcoal was added at the time of inoculation, are shown in Fig. 2. The effects of addition of charcoal 4 and 8 hr after inoculation are also shown. Evidently, the growth initiation process that is inhibited by charcoal requires between 4 and $8 \mathrm{hr}$ after inoculation. Delay of up to $8 \mathrm{hr}$ in the addition of charcoal had no consistent effect on elaboration of antigen.

Experiments with $\mathbf{F}$ mutants. Mutants that do not require adsorbent for optimal encapsulation

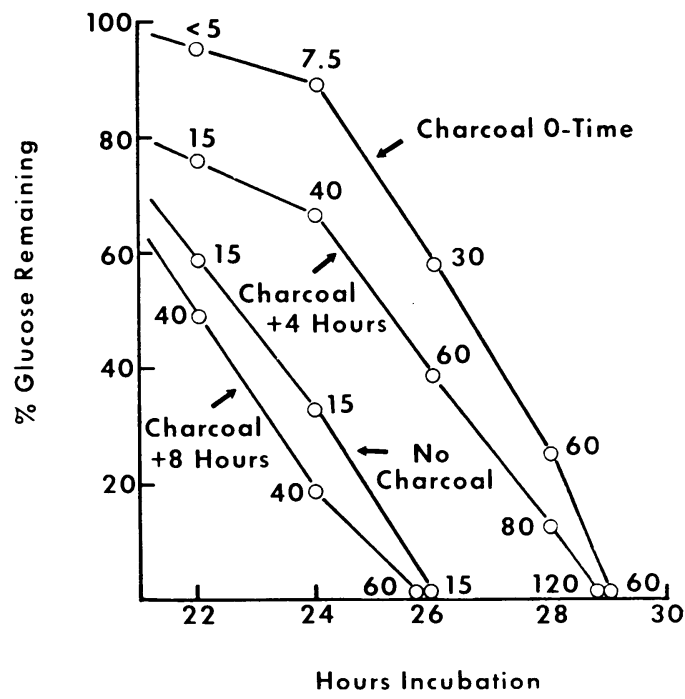

FIG. 2. Effect of time of addition of charcoal on glucose utilization and antigen elaboration in cultures grown from spore inocula. Numbers represent complement-fixing units per milliliter of culture. Curves represent cultures containing no charcoal, or containing $0.006 \%$ charcoal added after incubation for 0,4 , or 8 hr. 
have been described by Meynell and Meynell (9) and referred to as $F$ mutants. The biochemical changes associated with the mutation have not been elucidated. The nonencapsulated strain 116-NP2-F-R, derived from the F mutant of 116-NP2, was tested for its requirement for an adsorbent for antigen production. Typical results are shown in the last experiment in Table 2. The strain appeared to be no less dependent on adsorbent than the non-F strains tested previously.

\section{DISCUSSION}

Presence of an adsorbent represents an additional condition that stimulates significantly the elaboration of protective antigen, and represents another similarity between conditions for antigen elaboration and conditions for synthesis of polypeptide capsule by virulent strains. Evidence has not been obtained regarding the mechanism by which an adsorbent stimulates antigen elaboration.

Evidence was presented that adsorbent stimulates capsule synthesis by removal of a growth product, tentatively identified as fatty acid (8). Changes in the nature and distribution of cellular fatty acids are associated with alterations in the permeability of other microorganisms for amino acids and proteins (3). Control of protective antigen elaboration by bicarbonate also appears to be exerted through changes in cell permeability (10). Furthermore, a relationship between the actions of adsorbent and bicarbonate was indicated by the observation that addition of charcoal reduces the concentration of bicarbonate required for encapsulation (8). It may be proposed that the effect of adsorbent is exerted through alterations in cell permeability.

Because of the numerous parallels between capsule formation and antigen elaboration, the continued requirement for adsorbent of the strain derived from an $\mathrm{F}$ mutant was unexpected. However, the biochemical basis of the $F$ mutation is unknown, and its presence can be demonstrated only by its effect on encapsulation. Accordingly, there was no basis for determining whether the $F$ character was maintained in the nonencapsulated mutants used for antigen elaboration. It is also possible that the mechanism by which the $\mathrm{F} \mathrm{mu-}$ tation avoids the requirement for adsorbent is not applicable to the process of antigen elaboration.

The clear evidence that charcoal stimulates antigen elaboration only when phosphate is reduced indicates that charcoal replaces the high concentration of phosphate rather than acting by an independent mechanism. No evidence has been obtained of the nature of the adsorbent, the formation of which is postulated in the presence of $0.01 \mathrm{M}$ phosphate; presumably insoluble phosphates of metal ions are formed. It will be of interest to examine the effect of the addition of charcoal on the requirement for salts of calcium, magnesium, manganese, and iron in the medium. Presence of an optimal concentration of adsorbent may also reduce the requirement for bicarbonate.

The reduction of phosphate concentration, made possible by addition of charcoal, markedly increases the efficiency of the concentration and purification of protective antigen on DEAE cellulose columns. Observations on the isolation and properties of protective antigen will be presented in a subsequent paper.

\section{LITERATURE GITED}

1. Brachman, P. S., H. Gold, S. A. Plotkin, F. R. Fekety, M Werrin, and N. R. Ingraham. 1962. Field evaluation of a human anthrax vaccine. Amer. J. Pub. Health 52:632-645.

2. Darlow, H. M., F. C. Belton, and D. W. Henderson. 1956. The use of anthrax antigen to immunize man and monkey. Lancet 2:476-479.

3. Demain, A. L., and J. Birnbaum. 1968. Alteration of permeability for the release of metabolites from the microbial cell. Curr. Top. Microbiol. Immunol. 46:1-25.

4. Fiske, C. H., and Y. Subbarow. 1925. Colorimetric determination of phosphorus. J. Biol. Chem. 66:375-400.

5. Gruber, J., and G. G. Wright. 1967. Iodine-131 labeling of purified microbial antigens by microdiffusion. Proc. Soc. Exp. Biol. Med. 126:28-2284.

6. Lantos, J., and G. Ivanovics. 1964-5. Alkaline phosphatase repression by inorganic phosphate in Bacillus anthracis and Bacillus cereus. Acta Microbiol. Acad. Sci. Hung. 11:351355.

7. McGann, V. G., R. L. Stearman, and G. G. Wright. 1961. Studies on immunity in anthrax. VIII. Relationship of complement-fixing activity to protective activity of culture filtrates. J. Immunol. 86:458-464.

8. Meynell, E., and G. G. Meynell. 1964. The roles of serum and carbon dioxide in capsule formation by Bacillus anthracis. J. Gen. Microbiol. 34:153-164.

9. Meynell, G. G., and E. Meynell. 1966. The biosynthesis of poly D-glutamic acid, the capsular material of Bacillus anthracis. J. Gen. Microbiol. 43:119-138.

10. Puziss, M., and M. B. Howard. 1963. Studies on immunity in anthrax. XI. Control of cellular permeability by bicarbonate ion in relation to protective antigen elaboration. J. Bacteriol. 85:237-243.

11. Puziss, M., and G. G. Wright. 1959. Studies on immunity in anthrax. VII. Carbohydrate metabolism of Bacillus anthra$c i s$ in relation to elaboration of protective antigen. J. Bacteriol. 78:137-145.

12. Saifer, A., and S. Gerstenfeld. 1958. The photometric microdetermination of blood glucose with glucose oxidase. J. Lab. Clin. Med. 51:448-460.

13. Sergeant, T. P., C. E. Lankford, and R. W. Traxler. 1957. Initiation of growth of Bacillus species in a chemically defined medium. J. Bacteriol. 74:728-736.

14. Smith, H., D. W. Tempest, J. L. Stanley, P. W. Narris-Smith, and R. C. Gallop. 1956. The chemical basis of virulence in Bacillus anthracis. VII. Two components of the anthrax toxin; their relationship to known immunising aggressins. Brit. J. Exp. Pathol. 37:263-271.

15. Thorne, C. B. 1960 . Biochemical properties of virulent and 
avirulent strains of Bacillus anthracis. Ann. N.Y. Acad. Sci. 88:1024-1033.

16. Thorne, C. B., and F. C. Belton. 1957. An agar-diffusion method for titrating Bacillus anthracis immunizing antigen and its application to a study of antigen production. J. Gen. Microbiol. 17:505-516.

17. Wright, G. G., M. A. Hedberg, and J. B. Slein. 1954. Studies on immunity in anthrax. III. Elaboration of protective antigen in a chemically-defined non-protein medium. J. Immunol. 72:263-269.

18. Wright, G. G., M. Puziss, and W. B. Neely. 1962. Studies on immunity in anthrax. IX. Effect of variations in cultural conditions on elaboration of protective antigen by strains of Bacillus anthracis. J. Bacteriol. 83:515-522. 